\title{
Neutrino cross sections and nuclear structure
}

\author{
Giampaolo Co', Viviana De Donno, Chiara Maieron \\ Dipartimento di Fisica, Università del Salento and \\ Istituto Nazionale di Fisica Nucleare sez. di Lecce, \\ I-73100 Lecce, Italy
}

November 9, 2018

\begin{abstract}
The effects of the theoretical uncertainties in the description of the neutrino-nucleus cross sections for supernova neutrino energies are investigated.
\end{abstract}

The possibility of using neutrinos to make precision astrophysics measurements is not only related to our capability of detecting them, but also to the reliability of our theoretical description of the interaction between neutrinos and electrons and nuclei forming the detector. While the neutrino-electron cross section is well described in terms of perturbation theory, the situation becomes more complicated when nucleons and nuclei are involved. The internal structure of these systems is ruled by the strong interaction which is highly non perturbative in the energetic regime of interest for the neutrinos emitted from the stars.

We have investigated the consequences of the nuclear structure uncertainties on the neutrino-nucleus cross sections on the detection of supernova neutrinos. Computer simulations indicate that the fluences of the energies transported by these neutrinos, and antineutrinos, have thermal-like distributions, with average energies of about $15-30 \mathrm{MeV}$. The values of the temperature of the electron neutrinos and antineutrinos fluences are smaller than those characterizing $\mu$ and $\tau$ neutrinos, since, at the energies involved, only electron neutrinos, and antineutrinos, can interact with matter through charge current processes. 


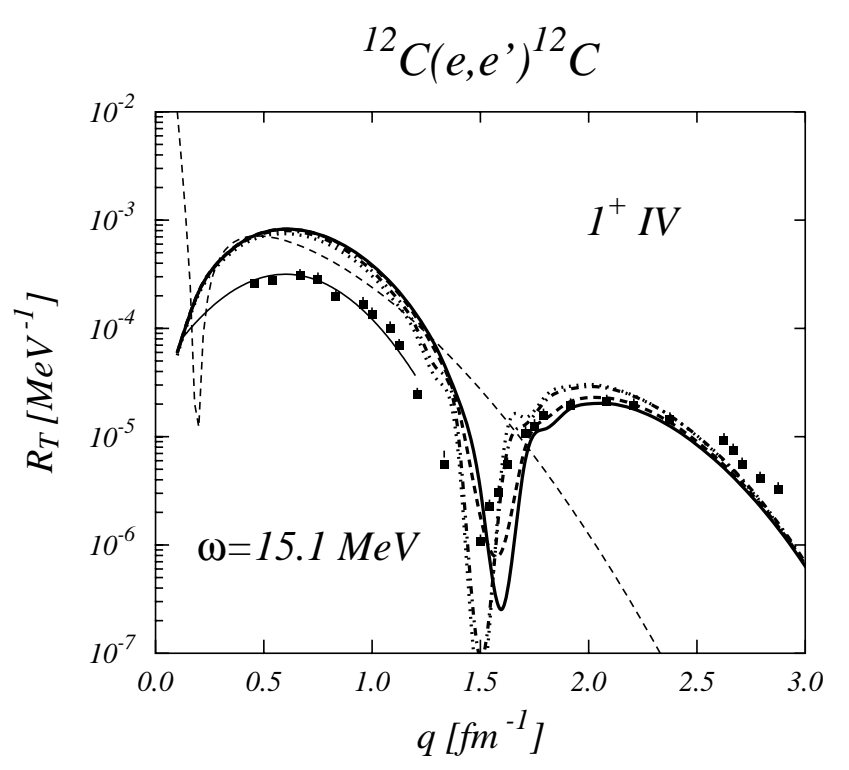

Figure 1: Electron scattering form factors for the isovector $1^{+}$state in ${ }^{12} \mathrm{C}$. The different thick lines have been obtained by using various effective interactions. The data are from Ref. [2]. The continuous thin line indicates our fit to the data. The dashed thin line shows the form factor used in Ref. [3].

We have studied the possibility of disentangling the temperatures of the $\mu$ and $\tau$ neutrinos and antineutrinos fluences from that of the electron neutrinos and antineutrinos. We have calculated neutrino-nucleus cross sections by using the effective theory called Random Phase Approximation (RPA) which describes the excited states of the nucleus as a linear combination of one-particle one-hole and one-hole one-particle excitations. The amplitudes of the linear combination are determined by solving a set of secular equations depending on the nucleon-nucleon effective interaction. We have constructed four effective interactions which reproduce with the same degree of accuracy the excitation energies of some specific states and some electromagnetic properties of the double magic nuclei ${ }^{12} \mathrm{C},{ }^{16} \mathrm{O},{ }^{40} \mathrm{Ca},{ }^{48} \mathrm{Ca},{ }^{90} \mathrm{Zr}$ and ${ }^{208} \mathrm{~Pb}$. A detailed description of the interactions and of the procedure used to construct them is given in [1]. Our study has been done by making RPA calculations with the four interactions and investigating the differences produced in various observables.

In our study we have observed a large sensitivity of the neutrino-nucleus cross section to the choice the residual interaction, when the nuclear ex- 
citation energy is above the nucleon emission threshold, as in the giant resonances region. For this reason, here, we address our attention to the excitation of discrete states, and specifically to the case of ${ }^{12} \mathrm{C}$ nucleus. We have calculated the ${ }^{12} \mathrm{C}\left(\nu, \nu^{\prime}\right){ }^{12} \mathrm{C}$ cross section for all the multipole excitations up to angular momentum $J=4$, and we have found that the main contribution is provided by the excitation of the isovector $1^{+}$state. This state belongs to a triplet of isovector $1^{+}$states. Two of them are the ground states of the ${ }^{12} \mathrm{~B}$ and ${ }^{12} \mathrm{~N}$ nuclei. These states can be reached from the ${ }^{12} \mathrm{C}$ ground state by means of the reactions ${ }^{12} \mathrm{C}\left(\bar{\nu}_{e}, e^{+}\right){ }^{12} \mathrm{~B}$ and ${ }^{12} \mathrm{C}\left(\nu_{e}, e^{-}\right){ }^{12} \mathrm{~N}$ which can be identified by measuring the electron and positron. Also the neutral current reaction exciting the $1^{+}$state can be identified by detecting the emitted photon having $15.1 \mathrm{MeV}$ energy.

In Fig. 1 we compare the results of our calculations with the inelastic scattering data of Ref. [2] for the isovector $1^{+}$state at $15.1 \mathrm{MeV}$. Since the transverse nuclear current matrix elements of the electron scattering cross sections are also present in the weak vector current contribution to the neutrino scattering cross section, we have taken them from experiment, by constructing momentum dependent quenching functions

$$
Q(q)=\frac{<J_{f}\left\|T_{J}^{e x p}(q)\right\| J_{i}>\left.\right|_{e, e^{\prime}} ^{2}}{\left|<J_{f} \| T_{J}^{V}(q)\right|\left|J_{i}>\right|_{R P A}^{2}},
$$

which we use to rescale the neutrino cross sections. This procedure has been applied below $q=1 \mathrm{fm}^{-1}$, i.e. in the kinematic region of interest for supernova neutrinos. In Fig. 1 we also show the form factor used in Ref. [3].

In Fig. 2 we show the cross sections for the charge exchange and charge conserving excitation of the $1^{+}$isovector triplet in ${ }^{12} \mathrm{C}$. The higher cross sections are those obtained in the bare RPA calculations, the set of lower cross section has been obtained after the rescaling procedure. The cross sections of Ref. [3] are the lowest ones. We have used these cross sections to estimate the expected number of events for the various neutrino interactions detected in the Large Volume Detector located at the Gran Sasso Laboratory [4. In our estimate we have used the following values for the supernova parameters: total energy transported by the neutrinos $E_{B} \sim 5.010^{52} \mathrm{erg}=$ $8.0110^{60} \mathrm{MeV}$, distance from the earth $\mathrm{D}=10 \mathrm{kpc}$, fraction of the energy carried by a specific type of neutrino, or antineutino, $f_{i}=1 / 6$. This last assumption implies that the energy is equally divided between the various neutrino types.

The total number of neutral current events $N_{N C}$ is obtained as the sum of the electron neutrino events calculated for a fluence with characteristic 

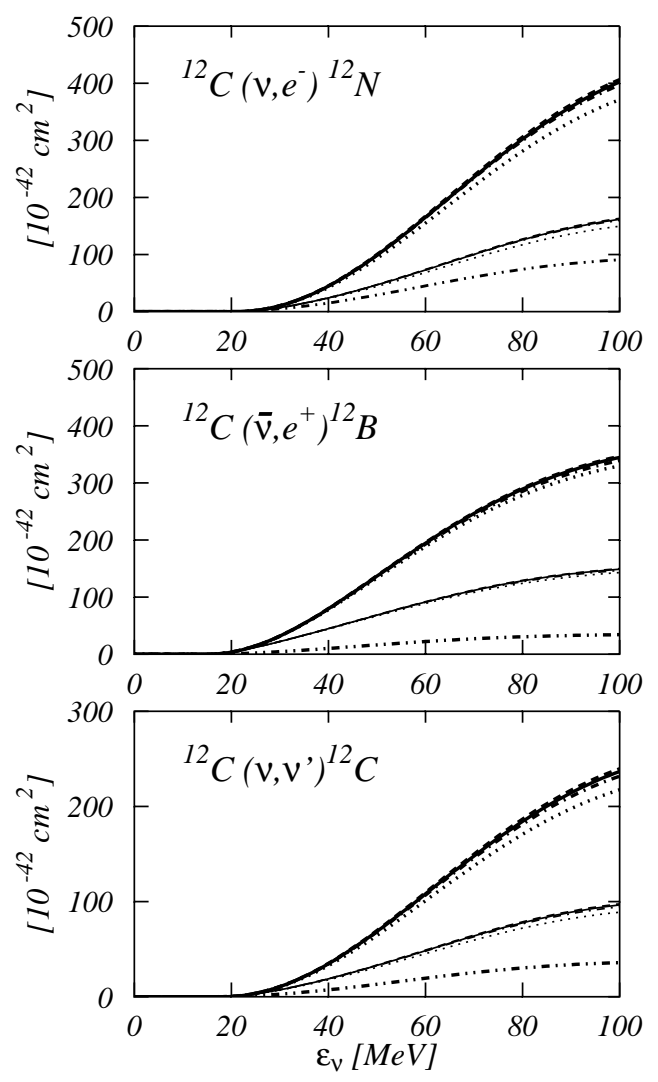

Figure 2: Neutrino cross sections for reactions exciting the isovector $1^{+}$triplet of states in ${ }^{12} \mathrm{C}$.

temperature of $4 \mathrm{MeV}$, plus the electron antineutrino having characteristic temperature of $5 \mathrm{MeV}$, plus the $\mu$ and $\tau$ neutrinos and antineutrinos events $N_{N C}^{T}$ having all the same temperature $T$.

The results of Fig. 2 indicate that there is a noticeable uncertainty on the size of the cross section, and consequently on the number of expected events. In order to reduce this uncertainty we consider the ratio $R=N_{N C}^{T} / N_{N C}$, shown in the upper panel of Fig. 3, as a function of $T$.

The upper and lower values of the bars shown in Fig. 3 indicate the largest and smaller value of $R$ calculated with our cross sections. The dots represent the values obtained with the cross sections of Ref. [3. The results of the upper panel of Fig. 3 show the stability of this new observable against 

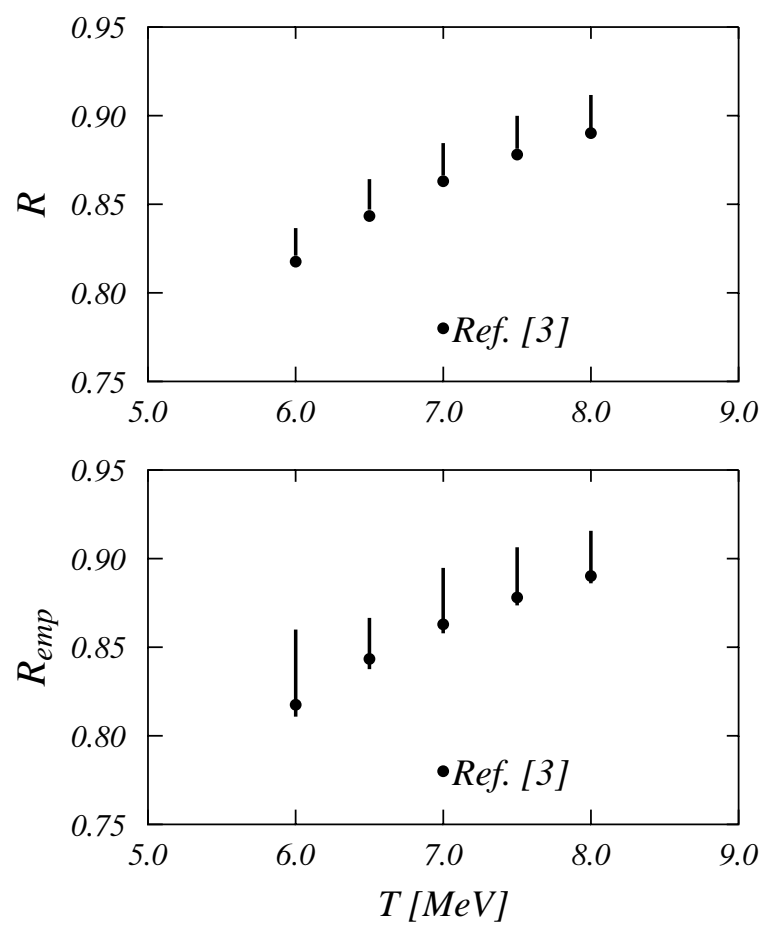

Figure 3: Upper panel. The ratio $R$ for some values of the characteristic temperature. Lower panel. The ratio $R_{e m p}$ of Eq. (2). The points in this case indicate the results one should obtain.

the nuclear structure uncertainties.

The experiment is not able to distinguish between neutral current events produced by electron neutrinos and antineutrinos and those induced by the other neutrinos and antineutrino types, but it has the information on the charge current events which can be induced only by electron neutrinos, and antineutrinos. We simulate an experimental case, by considering the count rates obtained with the cross sections of Ref. [3] as the detected counts $\mathrm{N}^{e m p}$. The ratio to be calculated is

$$
R_{e m p}=\frac{1}{N_{N C}^{e m p}}\left[N_{N C}^{e m p}\right.
$$




$$
\begin{gathered}
-N_{n p}^{e m p} \frac{\int_{e_{t h}}^{\infty} f(\epsilon) \sigma_{\nu_{e}, \nu_{e}^{\prime}}(\epsilon) d \epsilon}{\eta_{n p} \int_{e_{t h}}^{\infty} f(\epsilon) \sigma_{\nu_{e}, e^{-}}(\epsilon) d \epsilon} \\
\left.-N_{p n}^{e m p} \frac{\int_{e_{t h}}^{\infty} f(\epsilon) \sigma_{\bar{\nu}_{e}, \bar{\nu}_{e}^{\prime}}(\epsilon) d \epsilon}{\eta_{p n} \int_{e_{t h}}^{\infty} f(\epsilon) \sigma_{\bar{\nu}_{e}, e^{+}}(\epsilon) d \epsilon}\right] .
\end{gathered}
$$

The terms which multiply $N_{p n}^{e m p}$ and $N_{n p}^{e m p}$ depend on the knowledge of the neutrino-nucleus cross section, therefore $R_{e m p}$ is a model dependent quantity.

In the lower panel of Fig. 3 the spreading of the $R_{e m p}$ calculated with the various interactions and with the rescaled cross sections are indicated by the various bars. The correct values of $R_{e m p}$, obtained by inserting the cross sections of Ref. [3] in Eq. 2 are indicated by the dots. In conclusion, the uncertainty on the theoretical neutrino-nucleus cross sections in the giant resonance region is too large to make reliable predictions for astrophysical purposes. The discrete excitations are much better described, even though there are problems on the overall size of the cross sections. The use of ratio of observables reduces the effects of the nuclear structure uncertainties.

\section{References}

[1] V. De Donno, Nuclear excited states within the Random Phase Approximation theory, Ph.D. thesis, Università del Salento (Italy), unpublished (2008).

[2] Hicks, et al., Phys. Rev. C 30 (1984) 1.

[3] M. Fukugita, Y. Kohyama, K. Kubodera, Phys. Lett. B 212 (1988) 139.

[4] N. Y. Agafonova, et al., Astrop. Phys. 27 (2007) 254. 